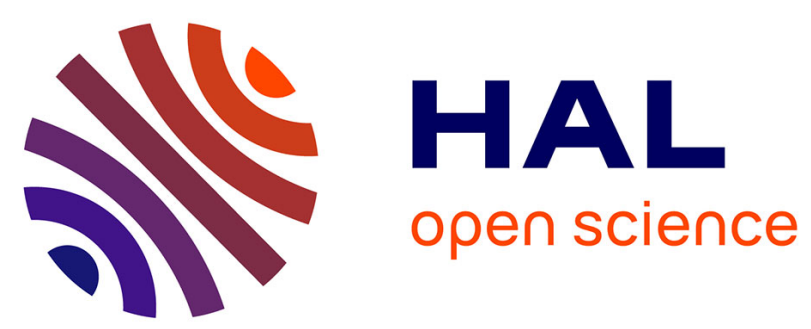

\title{
Limiter l'impact de la non-qualité sur la gestion des flux de production dans les ateliers à forts taux de reprises. Application au cas d'un fabricant de mobiliers laqués de très haute qualité
}

\author{
Mélanie Noyel, Emmanuel Zimmermann, Philippe Thomas, André Thomas, \\ Patrick Charpentier
}

\section{To cite this version:}

Mélanie Noyel, Emmanuel Zimmermann, Philippe Thomas, André Thomas, Patrick Charpentier. Limiter l'impact de la non-qualité sur la gestion des flux de production dans les ateliers à forts taux de reprises. Application au cas d'un fabricant de mobiliers laqués de très haute qualité. Journal Européen des Systèmes Automatisés (JESA), 2016, 49 (2), pp.179-198. 10.3166/JESA.49.179-198 . hal-01308813

\section{HAL Id: hal-01308813 \\ https://hal.science/hal-01308813}

Submitted on 28 Apr 2016

HAL is a multi-disciplinary open access archive for the deposit and dissemination of scientific research documents, whether they are published or not. The documents may come from teaching and research institutions in France or abroad, or from public or private research centers.
L'archive ouverte pluridisciplinaire HAL, est destinée au dépôt et à la diffusion de documents scientifiques de niveau recherche, publiés ou non, émanant des établissements d'enseignement et de recherche français ou étrangers, des laboratoires publics ou privés. 


\title{
Limiter l'impact de la non qualité sur la gestion des flux de production dans les ateliers à forts taux de reprises
}

\section{Application au cas d'un fabricant de mobiliers laqués de très haute qualité}

\author{
M. Noyel ${ }^{1,2,3}$, E. Zimmermann ${ }^{1,2,3}$, P. Thomas ${ }^{1,2}$, A. Thomas ${ }^{1,2}$, P. Charpentier ${ }^{1,2}$ \\ 1. Université de Lorraine, CRAN, UMR 7039, \\ Campus Sciences, BP 70239, 54506 Vandouvre-lès-Nancy cedex, France \\ philippe.thomas@univ-lorraine.fr, andré.thomas@univ-lorraine.fr. \\ patrick.charpentier@univ-lorraine.fr \\ 2. CNRS, CRAN, UMR7039, France \\ 3. Acta-Mobilier, \\ parc d'activité Macherin Auxerre Nord 89270 Monéteau \\ mnoyel@acta-mobilier.fr,ezimmermann@acta-mobilier.fr
}

RESUME. Le pilotage de la production est fortement dépendant des opérations relatives à la qualité des produits obtenus, car la non-qualité engendre des reprises qui vont fortement perturber les flux de production (boucles de retour, attentes, reprises, ...). Si la mise en place d'une approche de management de la qualité telle que la TQM est indispensable dans cette optique, elle est malheureusement bien souvent insuffisante. La méthodologie proposée dans cet article arrive en appui et complément d'une telle démarche et repose sur deux étapes principales. La première a pour but de limiter la non-qualité, et donc réduire les taux de reprises, en déterminant, en-ligne, le réglage optimal du processus en fonction des caractéristiques du lot considéré et de l'état de l'environnement. Ce réglage repose sur l'utilisation d'un modèle reliant les différents facteurs au risque d'occurrence des défauts générant une non-qualité. Ce modèle est construit par apprentissage à partir des données de production. Il est utilisé dans un deuxième temps pour simuler un plan d'expériences afin de déterminer les réglages optimaux des paramètres contrôlables. La deuxième étape consiste à évaluer l'impact des taux de reprises résiduels sur la perturbation des flux. Une combinaison d'indicateurs est proposée qui permet de mettre en évidence différentes zones de comportement de l'atelier, l'objectif étant d'associer une règle de pilotage optimale à chacune de ces zones. L'ensemble de l'approche est déployée sur un cas industriel dans l'entreprise Acta-Mobilier, fabricant de panneaux laqués haut de gamme pour les cuisinistes, stands, mobiliers de magasins....

ABSTRACT. Quality policy has an impact on the production flows control by the way of the reworks rate. The using of a quality management approach, as TQM, is useful but however often inadequate. The proposed approach must be seen as a supplement to such quality management. This approach is broken down into two steps. The goal of the first one is to reduce the reworks rates by tuning optimally and on line the process in function of the lot characteristics and the values of the environmental factors. This tuning uses a model linking the different influent factors with the default occurrence. This model is extracted from the production dataset by using a learning approach. It is used to simulate an experimental design in order to find the optimal tuning of the controllable parameters. The second step evaluates the impact of the residual reworks rates on the production flows. It uses a combination of different indicators in order to obtain a cartography of the considered workshop that highlights different workshop behavior areas. The final goal is to associate the good production control rule to each of these areas. All this approach is applied to the case of a manufacturer of high-finished lacquered panels.

MoTS-CLES : Reprises, perturbation des flux, indicateur, simulation, qualité, réseau de neurones, apprentissage, extraction de connaissance, cartographie.

KEYWORDS: Reworks, flows perturbation, indicator, simulation, quality, neural network, learning, knowledge extraction, cartography 


\section{Introduction}

L'augmentation des exigences clients en termes de performances et/ou de qualité de fabrication peut conduire les entreprises à se trouver dans des situations où elles travaillent à la limite technologique de leur outil de production ce qui conduit à gérer de forts taux de reprises. C'est le cas, en particulier, de l'entreprise Acta-Mobilier qui fabrique des panneaux de particules laqués de très haut de gamme pour des cuisinistes, ou pour réaliser des stands publicitaires pour de grands groupes participant à des salons. Le degré de qualité de surface requis pour ces panneaux conduit ainsi à des taux de reprises habituellement supérieur à $30 \%$ et pouvant même atteindre $80 \%$ pour certaines gammes de produits. Cette exigence client n'est pas toujours compensable par une évolution des processus de fabrication qui ne peut pas se faire à la même vitesse. L'objectif de ce papier est de présenter la procédure appliquée dans ce type d'entreprise pour « contrôler » ces taux de reprises de telle sorte à limiter leurs impacts sur le pilotage de la production. Bien évidemment, réduire l'impact des taux de reprises sur le pilotage de la production passe prioritairement par la réduction même de ces taux de reprises en assurant une meilleure maitrise de la qualité.

Actuellement, la famille de normes ISO 9000 (ISO 2015) définit le système de management de la qualité. Par ailleurs diverses politiques telles que, la qualité totale (Total Quality Management TQM) (Talib et Rahman 2012), le Juste à Temps (JàT) (Kannan et Tan 2005) où le Lean mangement (LM) (Vollmann et al. 1984) ont été proposées, qui toutes s'intéressent, de près ou de loin, à la notion de maîtrise de la qualité. En production, la qualité s'est toujours définie comme une conformité aux exigences (Crosby 1979), l'objectif étant de détecter et traiter toute déviation par rapport aux spécifications. Aussi, ces différentes politiques s'appuient sur, et exploitent, tout un ensemble d'outils (les 7 outils de base de la qualité) afin d'assurer la maitrise statistique des procédés : feuilles de relevés, logigramme, histogramme, diagramme d'Ishikawa, diagramme de corrélation, diagramme de Pareto, carte de contrôle. Ces outils, couramment utilisés dans le monde industriel, présentent le désavantage d'être «hors ligne », et donc pas suffisamment adaptés en présence d'un processus ou d'un environnement évolutif.

Si ces différents concepts sont indispensables à la maitrise de la qualité, et ont indéniablement un impact bénéfique sur les taux de reprise, ils sont malheureusement parfois insuffisants. Ainsi dans l'entreprise ActaMobilier la mise en œuvre de longue date de la philosophie LM n'a pas suffi pour réduire et maintenir les taux de reprises à un niveau acceptable. De plus, ces outils nécessitent d'attendre que la non qualité se produise afin de pouvoir détecter une dérive du procédé avant de pouvoir y remédier. Or, la qualité doit être contrôlée aussi près que possible de la source du défaut. Taguchi (1989) fut le premier à étudier la qualité en amont de la production du défaut par l'intermédiaire de la maîtrise des paramètres de contrôle du processus. Si les plans d'expériences peuvent être construits de telle sorte que les réglages des paramètres soient robustes à de telles évolutions, la notion de robustesse est bien souvent antinomique avec la notion d'optimalité, optimalité des réglages qui doit être recherchée lorsque l'outillage travaille à sa limite technologique pour atteindre le niveau de qualité recherché.

Dans l'objectif de limiter les taux de reprises et avec la même philosophie que celle proposée par Taguchi, nous allons chercher à déterminer le réglage optimal de l'outil de production en fonction des conditions d'utilisation. Pour ce faire, nous proposons de construire un modèle « liant » les paramètres de production avec le risque de génération de défauts par extraction des données de production. Pour ce faire, nous utilisons un perceptron multicouches tout à fait adapté à cette tache de classification. Ce modèle est ensuite utilisé dans un second temps pour déterminer le réglage optimal des paramètres contrôlables de l'outil de production en fonction de l'état des paramètres non contrôlables. La principale différence de philosophie entre notre approche et l'approche des plans d'expériences est que nous cherchons à déterminer le réglage optimal pour la situation d'exploitation «présente » et non pour toutes les «modalités » de réglage possible. L'exploitation d'une telle approche contribue grandement à la réduction des taux de reprises, mais ne parvient généralement pas à les supprimer totalement. L'étape suivante consiste donc à évaluer l'impact des taux de reprises résultants sur les flux de production pour en optimiser le pilotage dans un tel environnement perturbé.

Ces taux de reprises engendrent des boucles de production supplémentaires qui viennent compliquer le pilotage de la production (Colledani et Tolio 2006). La perturbation des flux due à la non-qualité n'a pas été étudiée en profondeur mais présente des comportements cumulatifs que l'on peut assimiler à un « effet boule de neige » (effet Forrester) qui la rendent difficile à évaluer comme à maîtriser. Dans le but de mesurer ce phénomène, un grand nombre d'indicateurs, tant de qualité que de contrôle des flux ont été proposés dans la littérature, mais qui pris individuellement, n'offrent tous qu' une vue partielle du problème (Noyel 2015). C'est pourquoi nous proposons 
de combiner différents indicateurs de non-qualité et de perturbation des flux qui offrent des vues différentes et complémentaires de l'état de l'atelier afin de réaliser une cartographie permettant de déterminer l'état courant de cet atelier.

Dans un deuxième temps, il est nécessaire de déterminer pour chaque état possible de l'atelier, les différentes actions à mener pour réduire l'impact des taux de reprises résiduels. Ces actions peuvent être, par exemple, des changements de règles de pilotage ou des renforcements des capacités de production (passage du $2 * 8$ au $3 * 8 \ldots$ )...

Le paragraphe suivant présentera le contexte et les enjeux de cette étude. Le paragraphe 3 portera sur la présentation de l'outil «en ligne » de management de la qualité, alors que le paragraphe 4 présentera lui les combinaisons d'indicateurs retenues conduisant à la réalisation d'une cartographie de l'état de l'atelier exploitable pour sélectionner les règles de pilotage. Le paragraphe 5 présentera les résultats obtenus avant de conclure.

\section{Contexte et enjeux}

Un problème de qualité en production va donc conduire à perturber la gamme de fabrication normale d'un produit de deux manières :

- Soit le problème qualité est réparable et un poste dédié à ces réparations existe, auquel cas le produit considéré est envoyé sur ce poste où il sera réparé. Il reprendra ensuite le cours normal de sa gamme de fabrication. Dans ce cas, une seule tâche effectuée sur le poste de réparation doit être adjointe à la gamme de fabrication normale et on parlera alors de « réparation ».

- Soit le problème qualité nécessite que le produit subisse à nouveaux plusieurs étapes de sa gamme normale de fabrication (éventuellement toutes si le défaut n'est pas réparable et nécessite la reproduction du produit). Auquel cas, le produit retourne à une étape précédente de sa gamme de fabrication et reprendra toutes les étapes qu'il avait déjà subies. La gamme modifiée contiendra alors deux séquences de tâches identiques mises en série et on parlera alors de « reprise».

Seul le cas des « reprises » est considéré ici car c'est le cas le plus préjudiciable au bon écoulement des flux de production. Le taux de reprises se mesure à la sortie d'un poste de travail lors du contrôle qualité. Les produits «dans les normes » poursuivent leur gamme de production normale alors que les produits « hors normes » voient leur gamme de fabrication modifiée. Le taux de reprises se rapporte donc à un poste de travail $M_{x}$ et est se définit par:

$$
T_{\text {reprise }}\left(M_{x}\right)=\frac{N b \text { de produits "hors normes" }}{N b \text { de produits travaillés sur } M_{x}}
$$

De nombreuses entreprises sont confrontées à ce type de problème, et comme nous l'avons dit, l'entreprise ACTA mobilier en fait partie. Parmi les 5 points de vue différents sur la qualité définis par Garvin (1984), le plus pertinent pour nous est «l'approche basée sur la fabrication» qui combine conformité aux exigences clients (mesure dans laquelle les caractéristiques de conception et d'exploitation d'un produit correspondent aux normes préétablies) et le «faire juste du premier coup » dans le but de réduire les coûts. La non-qualité s'exprime donc selon deux points de vue :

- Produit non-conforme aux exigences client,

- Produit conforme mais qui a nécessité une réparation pour l'être.

La non-qualité peut découler de processus de fabrication instables ou qui dérivent au cours du temps, ce qui générera une augmentation du taux des défauts. Dans ce cas, il est nécessaire de surveiller l'évolution de cette dérive (en utilisant des cartes de contrôle par exemple) et de la corriger quand elle ne devient plus tolérable. Elle peut être due également à la dispersion «naturelle » des processus lorsque ces derniers sont contraints de travailler à leurs limites technologiques. Dans ce cas, le taux de reprises sera fluctuant, et les perturbations engendrées sur les flux de production seront elles même fluctuantes et imprévisibles, rendant tout pilotage de production aléatoire. Obtenir un pilotage de la production performant passe donc obligatoirement par une bonne maitrise de la qualité.

\section{Management en ligne de la qualité}

\subsection{Comment contrôler la qualité}

Comme expliqué dans l'introduction, les philosophies qui s'intéressent à la maitrise de la qualité telles que la TQM, le LM, ou le JàT et qui s'appuient en particulier sur les 7 outils de base de la qualité sont « hors ligne » et nécessitent d'attendre la génération de défauts pour qu'ils puissent être détectés avant d'en rechercher et traiter la 
ou les causes. Si l'exploitation des plans d'expériences, qui a pour but de prévenir l'apparition du défaut en recherchant le réglage optimal du processus, permet de répondre en partie à cette limitation, elle reste insuffisante en présence d'un processus ou d'un environnement évolutifs et ce, même si ces plans d'expériences sont construits de manière à viser la qualité de robustesse. L'ensemble de ces approches et outils sont donc utiles, voir incontournables, mais insuffisants. Une classification des facteurs influents en fonction de leur contrôlabilité a été proposée (Noyel et al 2013), le parallèle étant fait avec la classification d'Ishikawa :

- Facteurs environnementaux (Milieu) tels que température ou humidité. Ces facteurs sont souvent peu ou noncontrôlables bien qu'ils soient aisés à mesurer.

- Facteurs techniques (Machine - Méthode) résultent de l'état des machines durant les opérations. Ils sont contrôlables lorsqu'ils correspondent aux réglages machines.

- Facteurs humains (Main d'œuvre). Ces facteurs sont difficiles à prendre en compte car ils varient fortement en fonction des opérateurs. Leur contrôle passe par l'établissement de standard ou de poka yoke qui présentent des limitations et des contraintes.

C'est le caractère évolutif des facteurs environnementaux (non contrôlables) et éventuellement des facteurs humains qui rendent l'utilisation d'un réglage unique des paramètres contrôlables, quelques soient les conditions, insuffisant, même si ce réglage a été optimisé à l'aide d'un plan d'expériences. Notre objectif est au contraire de faire évoluer le réglage des paramètres contrôlables en fonction de l'évolution des facteurs non contrôlables afin de disposer en permanence du réglage optimal du processus dans le but de limiter la génération de défauts. Pour ce faire nous avons besoin de disposer d'un modèle permettant de relier l'évolution des différents facteurs (contrôlables et non contrôlables) au risque de génération de défauts. Ce modèle, nous proposons de l'extraire directement des données de production en exploitant des techniques d'apprentissage.

\subsection{Extraction de connaissance à partir de données}

L'idée est donc d'extraire le modèle désiré directement des données de production. Les besoins de traçabilité notamment ont poussé la plupart des entreprises à mettre en œuvre des outils de contrôle de la production permettant de collecter aussi bien les données de production que les données produit, et ce de manière automatisée ou semi automatisée. Il est donc tout à fait possible de mettre en place un processus d'extraction de connaissance (Knowledge Discovery in Data - KDD) à partir des données de production pour identifier les conditions de génération de défauts. Le cœur de ce processus KDD est le data mining qui peut être réalisé en exploitant une grande diversité d'outils et ce, pour réaliser différents objectifs dont les principaux sont :

- Classification : regrouper les données en différents groupes ou classes prédéfinis

- Régression : mapping des données d'un espace d'entrée vers un espace de sortie

- Clustering : regrouper les données similaires.

Nous nous trouvons en présence d'un problème de surveillance de la qualité. L'objectif de notre modèle est donc d'être capable de répartir les données en deux classes : défaut et non défaut. Nous sommes donc bien en présence d'un problème classique de classification supervisée.

Le processus KDD complet s'effectue en plusieurs étapes (Patel et Panchal 2012) :

- Sélection : collecte des données à partir de diverses sources.

- Préprocessing : nettoyage des données

- Transformation : conversion à un format commun, transformation à un nouveau format

- Data mining : obtention des résultats désirés

- Interprétation/Evaluation/Présentation

\subsubsection{Sélection, preprocessing et transformation}

Par définition même, sans donnée pas de processus KDD. Collecter les données constitue donc une tâche primordiale dans un tel processus. Il est nécessaire de déterminer l'ensemble des facteurs (contrôlables et non contrôlables) susceptibles d'influer sur la production de défauts. Cette étape peut s'effectuer par interview d'experts et dans le doute, il est préférable de considérer qu'un facteur est susceptible d'avoir un impact plutôt que 
le contraire. Il est nécessaire d'être le plus exhaustif possible lors de cette phase et d'utiliser des techniques de sélection de variables lors de la phase de data mining qui permettra d'éliminer les facteurs non significatifs.

Une fois la liste de ces facteurs potentiellement influents dressée, il est nécessaire de collecter les valeurs de ces différents facteurs qui influencent la qualité en cours de production, l'objectif étant d'éviter d'avoir à réaliser des expériences particulières comme pour un plan d'expériences. La collecte de données doit s'effectuer à deux niveaux :

- En amont du poste de travail afin de collecter les conditions de production et les caractéristiques produit ainsi que les facteurs environnementaux (l'ensemble des facteurs contrôlables et non contrôlables précédemment listés),

- En aval du poste de travail pour relever les défauts générés.

Il est utile de rendre cette étape de collecte de données la plus automatisée possible dans le but d'en limiter le coût mais aussi et surtout de limiter le nombre de valeurs aberrantes inhérent à la saisie manuelle. Il est notable cependant que dans bien des cas, la collecte des défauts qualité reste une tâche manuelle, sujette aux erreurs humaines ainsi qu'à la subjectivité de l'opérateur.

Les données collectées doivent être «préprocessées » afin, par exemple, de synchroniser les différentes bases de données, supprimer les valeurs aberrantes évidentes, digitaliser les données qualitatives comme des couleurs...

\subsubsection{Data mining et évaluation}

Le volume de données à analyser est souvent important (Agard et Kusiak 2005). La plupart des entreprises collectent et archivent un grand nombre de données à des fins principalement de traçabilité mais exploitent rarement ces données autrement que pour réaliser des indicateurs pour le management temps réel de la production.

L'objectif est d'exploiter ces données sous employées afin d'en extraire de la connaissance concernant la génération de défauts. Pour ce faire, différents outils peuvent être utilisés tels que les réseaux Bayésiens, les arbres de décision, les Support Vector Machines (SVM), les réseaux de neurones (NN)... Les arbres de décisions permettent d'obtenir une classification rapide mais sont très sensibles aux données bruitées (Patel et Panchal 2012). Les réseaux Bayésiens sont dédiés à l'exploitation de données discrètes et nécessitent une phase de discrétisation en présence de données continues susceptibles de faire perdre de l'information. SVM et NN utilisent des concepts très proches et conduisent à des résultats très similaires. Parfois les SVM donnent de meilleurs résultats (Meyer et al. 2003), parfois ce sont les réseaux de neurones (Hajek et Olej 2010, Paliwal et Kumar 2009). Quatre outils ont été testés et comparés par Noyel (2015) sur les données d'ACTA mobilier : les SVM, le perceptron multicouches (MLP), les arbres de décisions et les k plus proches voisins). Nous ne présenterons ici que les MLP qui ont donné les meilleurs résultats lors de notre étude.

Le MLP est un réseau de neurones particuliers qui effectue une recherche locale de minimum. Ce fait induit que différents jeux de paramètres initiaux doivent être utilisés pour l'apprentissage afin d'éviter le risque de tomber dans un minimum local très éloigné du minimum global recherché. Ces différents apprentissages sur des jeux de poids initiaux différents conduisent à des classificateurs différents plus ou moins performants pouvant être exploités dans un ensemble classificateur (Thomas et al. 2013) qui ne sera pas considéré ici.

La structure d'un tel MLP est donnée par :

$$
z=g_{2}\left(\sum_{i=1}^{n_{1}} w_{i}^{2} \cdot g_{1}\left(\sum_{h=1}^{n_{0}} w_{i h}^{1} \cdot x_{h}^{0}+b_{i}^{1}\right)+b\right)
$$

où $x_{h}^{0}$ sont les $n_{0}$ inputs du réseau, $w_{i h}^{1}$ sont les poids connectant la couche d'entrée à la couche cachée, $b_{i}^{1}$ sont les biais des neurones de la couche cachée, $g_{1}($.$) est la fonction d'activation des neurones cachés (ici la tangente$ hyperbolique), $w_{i}^{2}$ sont les poids connectant les neurones cachés au neurone de sortie, $b$ est le biais du neurone de sortie et $g_{2}($.$) est la fonction d'activation de neurone de sortie (ici une sigmoïde car nous sommes en présence d'un$ problème de classification) et $z$ est la sortie du réseau.

La base de données collectée est subdivisée en deux parties. La première est utilisée pour déterminer les paramètres du modèle. La deuxième a pour but de valider le modèle en testant ses capacités de généralisation. La détermination des paramètres d'un MLP s'effectue en trois étapes : initialisation, apprentissage et pruning.

L'initialisation est importante car l'apprentissage d'un MLP effectue une recherche locale de minimum. Aussi, un mauvais jeu de paramètres initiaux peut conduire à tomber dans un minimum local très éloigné du minimum global. Pour éviter cela, différents jeux de paramètres initiaux doivent être construits afin de permettre à l'apprentissage de commencer dans différentes zones du domaine du critère. Différents algorithmes d'initialisation 
ont été proposés (Thomas et Bloch 1997). L'algorithme utilisé ici est celui proposé par Nguyen et Widrow (1990) qui permet d'associer une initialisation aléatoire des paramètres à un placement optimal dans l'espace des entrées.

L'apprentissage a pour but de faire correspondre la sortie du réseau avec les données. A nouveau, différents algorithmes d'apprentissage ont été proposés dans la littérature. L'algorithme retenu est l'algorithme de Levenberg-Marquard qui permet d'associer la rapidité des méthodes du Hessien à la stabilité des méthodes du gradient. Dans les applications industrielles, les données sont bruitées et polluées par de nombreuses valeurs aberrantes. Afin de limiter l'impact de ces valeurs aberrantes sur le résultat, un critère robuste à la présence de valeurs aberrantes est exploité en conjonction avec l'algorithme de Levenberg-Marquard (Thomas et al. 1999). L'utilisation d'un critère robuste a également un effet de régularisation qui permet de prévenir le problème de surapprentissage.

Un point important lors de la construction d'un modèle neuronal est la détermination de la structure du réseau. Ceci est particulièrement vrai dans le cas où, lors de la collecte de données, aucune connaissance experte n'est disponible pour déterminer quels facteurs influent sur la génération de défauts. Dans ce cas, il est nécessaire à cette étape d'éliminer des entrées inutiles. Pour ce faire, le pruning, exploite une structure surdimensionnée et élimine les paramètres les moins significatifs dans un deuxième temps (Engelbrecht 2001, Setiono et Leow 2000). Nous utiliserons une approche de pruning qui présente l'avantage de sélectionner simultanément le nombre de neurones cachés et les neurones d'entrée. L'algorithme de pruning utilisé est celui proposé par Thomas et Suhner (2015) qui fournit un bon compromis entre rapidité et efficacité.

\subsubsection{Réglage optimal des paramètres contrôlables}

Le modèle classificateur ainsi constitué permet de relier les différents facteurs influant à la génération de défaut. Il est donc possible de l'exploiter pour déterminer les valeurs des paramètres contrôlables (facteurs techniques) en fonction de l'état des facteurs non contrôlables (environnementaux et humains) pour limiter les risques de production de défaut.

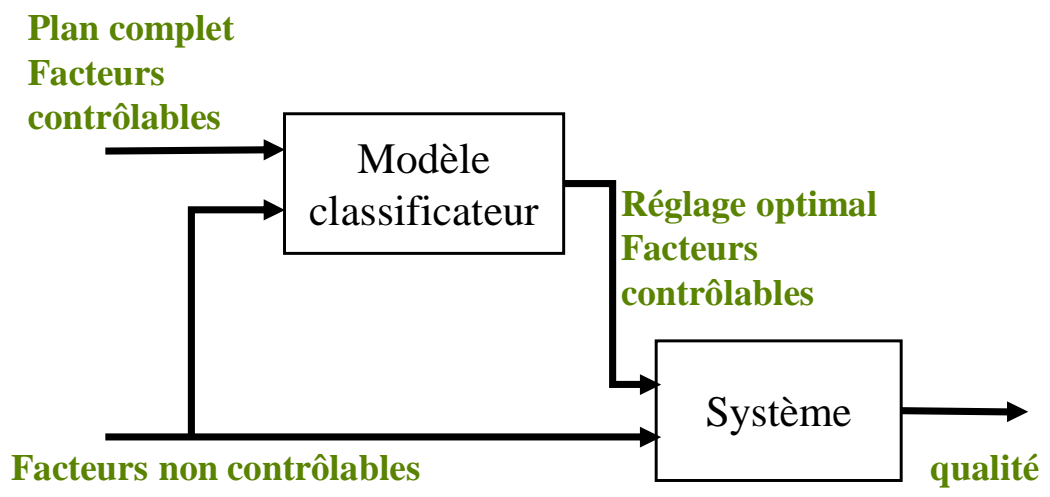

Figure 1. Réglage optimal des facteurs contrôlables

L'idée, schématisée figure 1, est de figer les facteurs non contrôlables aux valeurs correspondant à l'état courant du processus et de simuler un plan d'expériences complet pour les facteurs contrôlables. Il est alors possible d'étudier l'impact des différentes valeurs de ces facteurs contrôlables sur le risque de production de défauts et donc, d'en trouver les valeurs optimales de réglage. Une telle approche permet de disposer en permanence des réglages optimaux du processus ce qui permet de réduire les taux de reprises.

L'approche proposée permet de limiter les taux de reprises sans généralement parvenir à totalement les supprimer. Aussi est-il nécessaire de prendre en compte ces taux de reprises résiduels pour le pilotage de la production. 


\section{Impact des taux de reprises sur les flux}

Piloter efficacement la production passe par le maintien de bas taux de reprises. Cependant, même en diminuant et en stabilisant ces taux, les perturbations résultantes sur les flux de produits restent conséquentes et leurs phénomènes cumulatifs rendent la planification très difficile, voire impossible. Les produits défectueux désorganisent les lots de fabrication (division, regroupage... problématique du Burbidge Effect) contribuent à modifier les gammes (étapes de réparation supplémentaires qui surchargent les plannings de travail... problématique du Houlihan Effect) ou à changer les priorités d'ordonnancement (Disney et Towill 2003). La nonqualité est donc une des causes primaire du bullwhip effect.

Tableau 1. Indicateurs de non qualité de flux

\begin{tabular}{|c|c|}
\hline Indicateur & Vue et disponibilité \\
\hline $\begin{array}{l}N_{\text {config }} \text { ou } N_{\text {défaut }} \text { (équivalent dans } \\
\text { le cas de lot unitaire) : Pourcentage } \\
\text { de pièces qui ont présenté au moins } \\
\text { un défaut. }\end{array}$ & $\begin{array}{l}\text { Capacité à réaliser le produit juste du premier } \\
\text { coup. } \\
\text { Disponible sur une période donnée si les } \\
\text { défauts sont relevés dès leur création à chaque } \\
\text { poste susceptible de générer des défauts. }\end{array}$ \\
\hline $\begin{array}{l}R O_{E T}: \text { Le Ratio d'Opération RO } \\
\text { correspond au nb d'opérations } \\
\text { réalisées par rapport à ce qui était } \\
\text { prévu dans la gamme. Comme il y a } \\
\text { une valeur par pièce/lot, il convient } \\
\text { de prendre la moyenne et/ou l'écart } \\
\text { type. }\end{array}$ & $\begin{array}{l}\text { Capacité de réparer correctement le produit } \\
\text { défectueux ou la prévisibilité du respect des } \\
\text { délais. } \\
\text { Disponible a posteriori par comparaison avec } \\
\text { la gamme de fabrication prévue si la } \\
\text { traçabilité des produits est correctement } \\
\text { sauvegardée }\end{array}$ \\
\hline $\begin{array}{l}N_{\text {travail }} \text { ou } R O_{\text {moy }} \text { : Pourcentage de } \\
\text { pièces effectivement travaillées sur } \\
\text { la machine }\end{array}$ & $\begin{array}{l}\text { Densité du flux ou charge de travail réelle sur } \\
\text { l'atelier } \\
\text { Facilement disponible par poste pour } N_{\text {travail }} \\
\text { mais disponible plus difficilement et a } \\
\text { posteriori pour } R O_{\text {moy }}\end{array}$ \\
\hline
\end{tabular}

Le lien entre non-qualité (ou reprises) et perturbation des flux n'a pas toujours été bien mis en évidence. Cependant, dès les années 50, on parle de non-qualité avec la méthode COQ (Cost of quality) et l'usine fantôme qui est un atelier parallèle à l'atelier officiel dont la mission est de réparer les défauts de l'usine officielle (Feigenbaum 1951). La première norme sur le sujet apparaît en France en 1986 (X 50-126) pour évaluer les coûts de non-qualité (Abouzahir et al. 2003). La perturbation des flux de production par les reprises est particulièrement importante si les reprises ont lieu en aval du processus de production considéré (Love et al. 1999).

Divers indicateurs ont été proposés afin d'évaluer aussi bien la non-qualité que la perturbation des flux. Cependant, chaque indicateur pris individuellement va donner une vision partiale et partielle du problème. L'idée est donc de développer un système d'indicateurs synthétique donnant une vision globale en temps réel de l'état de l'atelier, de l'impact des reprises et/ou de la non qualité sur la fluidité (qualité) des flux.

De nombreux indicateurs ont été définis pour évaluer la non-qualité ainsi que la perturbation des flux (Noyel et al. 2014). Les tableaux 1 et 2 présentent respectivement, et sans être exhaustifs, les principaux indicateurs relatifs à la non-qualité et ceux relatifs à la perturbation des flux. Comme tous les indicateurs de performance, ces indicateurs correspondent à des triplets (objectif, mesure, variable) qui exprime l'efficience d'un système par rapport à une norme préétablie (AFGI 1992).

Tableau 2. Indicateurs de perturbation des flux

\begin{tabular}{|l|l|}
\hline Indicateur & Vue et disponibilité \\
\hline $\begin{array}{l}N_{E C} \text { et } N_{\text {stock }}: \mathrm{Nb} \text { de pièces en } \\
\text { en-cours global et niveau de stock } \\
\text { en amont d'un poste de charge }\end{array}$ & $\begin{array}{l}\text { Densité du flux ou charge de travail réelle sur } \\
\text { l'atelier }\end{array}$ \\
& $N_{E C}$ disponible en temps réel en tant que \\
& valeur offerte traditionnellement par les ERP. \\
& $N_{\text {stock disponible de la même manière ou }}$ \\
& mesurable directement en amont des postes de \\
& travail critiques. \\
\hline
\end{tabular}




\begin{tabular}{|l|l|}
\hline $\begin{array}{l}C_{\max } \text { et } T_{A}: \text { Temps pour terminer } \\
\text { la fabrication de toutes les pièces à } \\
\text { produire et temps moyen d'attente } \\
\text { des pièces }\end{array}$ & $\begin{array}{l}\text { Vitesse d'écoulement du flux } \\
C_{\max } \text { disponible a posteriori, une fois que } \\
\text { toute la production a été réalisée ou estimable } \\
\text { mais avec beaucoup de dispersion. } T_{A} \\
\text { disponible plus facilement sur le terrain (ex : } \\
\text { mise en place de minuteurs). }\end{array}$ \\
\hline$R_{\max }:$ Retard maxi & $\begin{array}{l}\text { Ponctualité } \\
\text { Disponible a posteriori puisque le lot doit être } \\
\text { terminé pour pouvoir évaluer son retard par } \\
\text { rapport à une date d'expédition prévue. }\end{array}$ \\
\hline$N_{\text {retard }}: \%$ de retards. & $\begin{array}{l}\text { Satisfaction client } \\
\text { Disponible a posteriori pour la même raison } \\
\text { que } R_{\text {max }}\end{array}$ \\
\hline
\end{tabular}

Dans un premier temps, nous pouvons étudier le comportement de ces indicateurs en fonction de l'augmentation progressive du taux de reprises. La perturbation des flux reste une notion compliquée à mesurer. Chaque indicateur offre une seule vue du problème. Pour en avoir un aperçu plus global et donc plus réaliste, il devient important de travailler sur une combinaison de ces indicateurs.

Par ailleurs, il est possible d'étudier l'évolution de chacun de ces indicateurs lorsque le taux de reprises augmente et ce, pour différentes règles de pilotage. Un exemple en est donné figure 2. Il montre l'évolution du nombre de retards $\mathrm{N}_{\text {retard }}$ en fonction de l'évolution du taux de reprises pour deux règles de pilotage classique de production différentes : EDD et FIFO. Ces résultats sont obtenus par simulation à l'aide du modèle simplifié Arena ${ }^{\circledR}$ d'un atelier sur lequel on peut faire varier les taux de reprises et les règles de pilotage.

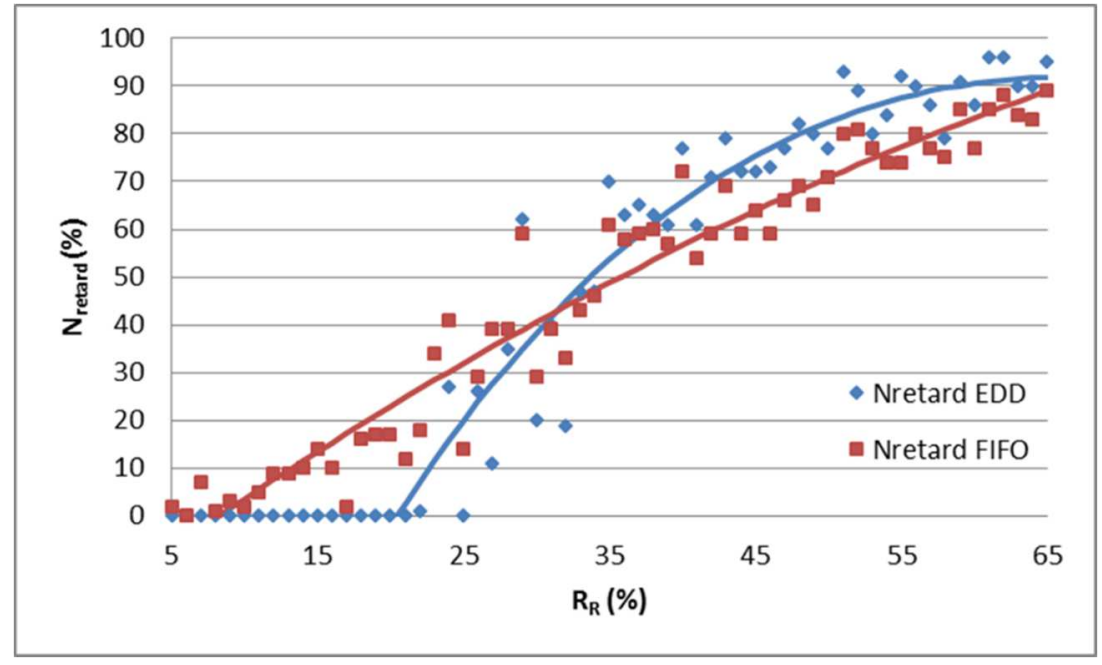

Figure 2. Règles d'utilisation des lois de pilotage EDD et FIFO

D'après cet exemple il apparait que pour la maîtrise du nombre de retards il est judicieux d'utiliser la règle EDD tant que le taux de reprise reste maitrisé en deçà d'un certain seuil (environ $31 \%$ dans l'exemple) mais qu'il est préférable de basculer sur une règle de pilotage de type FIFO lorsque le taux de reprise dépasse ce seuil.

Cependant, comme expliqué précédemment n'utiliser qu'un seul indicateur risque de donner une vision biaisée du problème. Aussi semble-t-il judicieux de combiner deux ou plus de ces indicateurs afin d'obtenir une vision plus globale de l'état de l'atelier. Par exemple, l'indicateur $N_{\text {défaut }}$ évalue le nombre de pièces ayant présenté au moins un défaut. Cet indicateur résulte directement des méthodes de contrôle de la qualité et est couramment utilisé dans les entreprises. Il permet de représenter la capacité de l'atelier à réaliser le produit « juste » du premier coup. 
Cependant, il ne permet pas de comprendre et prévoir l'impact sur la perturbation des flux. Par contre, si on combine cet indicateur avec un indicateur de perturbation des flux, par exemple $N_{\text {retard }}$, il est possible de mettre en évidence différents seuils déterminant des zones dans lesquelles la perturbation des flux devient trop importante pour être maitrisée.

A partir de ces seuils, une cartographie peut être établie décrivant l'état de l'atelier en fonction de la valeur de ces différents indicateurs. Dans cette cartographie, des zones de comportement peuvent être construites en fonction des différents seuils définis.

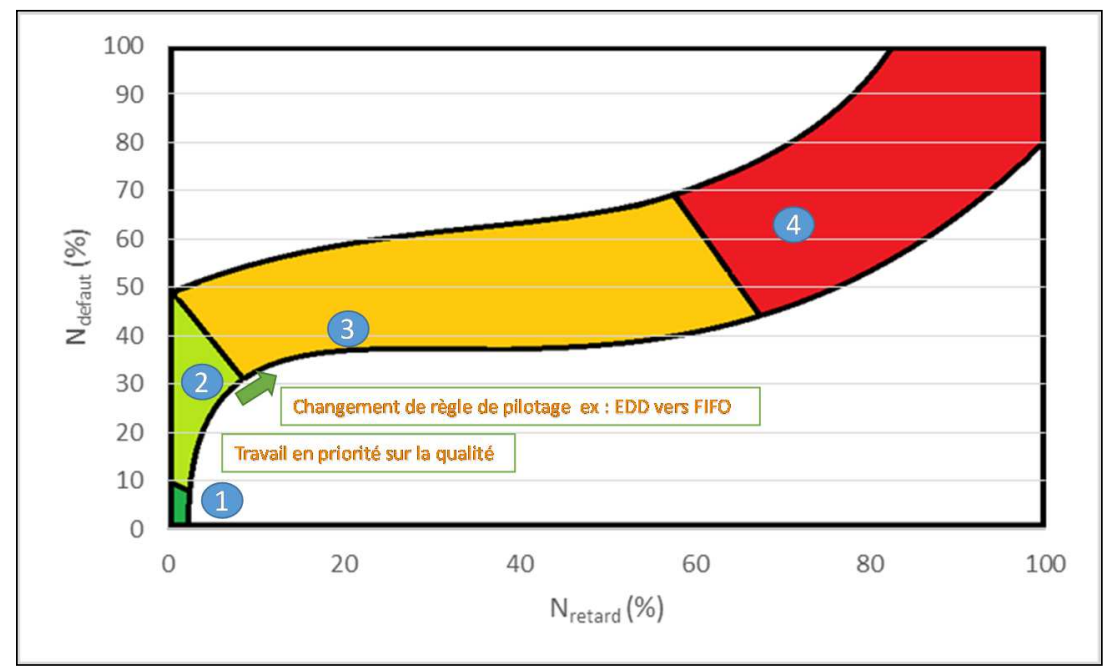

Figure 3. Règles d'utilisation des lois de pilotage EDD et FIFO

La figure 3 présente un exemple d'état d'atelier construit à partir de la combinaison des deux indicateurs $N_{\text {défaut }}$ et $N_{\text {retard }}$. Les différents seuils permettent de définir 4 zones. La zone 1 représente l'état de l'atelier ou la qualité est totalement maitrisée. La zone 2 correspond à une zone où l'augmentation de défauts n'entraine pas de retards supplémentaires et correspond donc à une zone d'absorption (les capacités de production sont suffisantes pour empêcher que les défauts générés ne perturbent suffisamment les flux pour entrainer des retards). La zone 3 représente l'état de l'atelier où quelques défauts supplémentaires font exploser le nombre de retards. On a défini cette zone comme une zone de dérive. Enfin, la zone 4 représente une zone de saturation.

Dans un deuxième temps, il est nécessaire de déterminer pour chaque zone les différentes actions à mener pour réduire l'impact des taux de reprises résiduels. Ces actions peuvent être, par exemple, des changements de règles de pilotage, des renforcements des capacités de production (passage du $2 * 8$ au $3 * 8 \ldots$ )...

Ainsi, le passage d'une zone à une autre implique un changement dans le pilotage de l'atelier pour que ce dernier reste optimal. Par exemple, dans la cartographie de la figure 3, le passage de la zone 1 à la zone 2 peut impliquer de lancer un chantier qualité alors que le passage de la zone 2 à la zone 3 peut, lui, impliquer de changer de règle de pilotage. La détermination de la règle optimale à associer à chaque zone peut être réalisée à l'aide d'un plan d'expériences.

\section{Cas d'étude}

\subsection{Présentation de l'entreprise}

L'entreprise Acta-Mobilier produit des panneaux de MDF laqués de haute qualité pour des cuisinistes, des stands ou des mobiliers de magasin ou de bureau... Dans sa quête d'amélioration continue, l'entreprise est certifiée ISO 9001, ISO 14001, OHSAS 18001 et met en place un processus Kaizen (5S) ; rappelons que l'un des points du Kaizen concerne la maitrise de la qualité.

L'entreprise est subdivisée en 5 ateliers qui sont tous susceptibles d'être sources de défauts. Ce travail se focalise sur un des ateliers articulé autour d'un robot de laquage. Bien que cet atelier soit totalement automatisé et qu'il ne subit donc pas l'impact de facteurs humains, la qualité de production est très variable et peut varier de 
$10 \%$ à $45 \%$ d'un jour sur l'autre sans changement de réglage et malgré cette mise en place d'outils de l'amélioration continue.

\subsection{Présentation du process et du modèle}

Le système considéré est donc un robot de laquage. La liste des facteurs influant sur la production de défauts a été obtenue par interview d'experts.

Les facteurs techniques collectés sont : taux de remplissage, nombre de passes, temps par table, litres par table, grammage, nombre de couches, nombre de pièces, et temps de séchage. Ces facteurs techniques doivent être subdivisés en facteurs contrôlables et non contrôlables (imposés par la gamme produit). Les facteurs contrôlables sont le taux de remplissage, le grammage et le temps de séchage.

Outre ces facteurs techniques, trois facteurs environnementaux (non contrôlables) sont également collectés : température, pression, humidité.

A la sortie du robot, une phase de collecte manuelle des défauts est effectuée. 30 types de défauts différents sont relevés. Cette étude se concentre sur un défaut particulier, la présence de grain.

\subsection{Surveillance « en ligne » de la qualité}

La base de données collectées comporte 2270 échantillons. Ce jeu de données est subdivisé aléatoirement en 2 jeux, un pour l'apprentissage (1202) et un pour la validation (1068). La structure initiale du réseau comporte 15 neurones d'entrée, 25 cachés et 1 de sortie. Afin de palier au risque d'obtention de minimum local très éloigné du minimum global, 100 jeux de poids initiaux ont été créés donnant lieu à l'obtention de 100 modèles différents après apprentissage et pruning. Le modèle sélectionné est celui donnant les meilleurs résultats sur le jeu de validation. Pour ce modèle, la phase de pruning a conduit à l'élimination de 6 neurones cachés et une entrée.
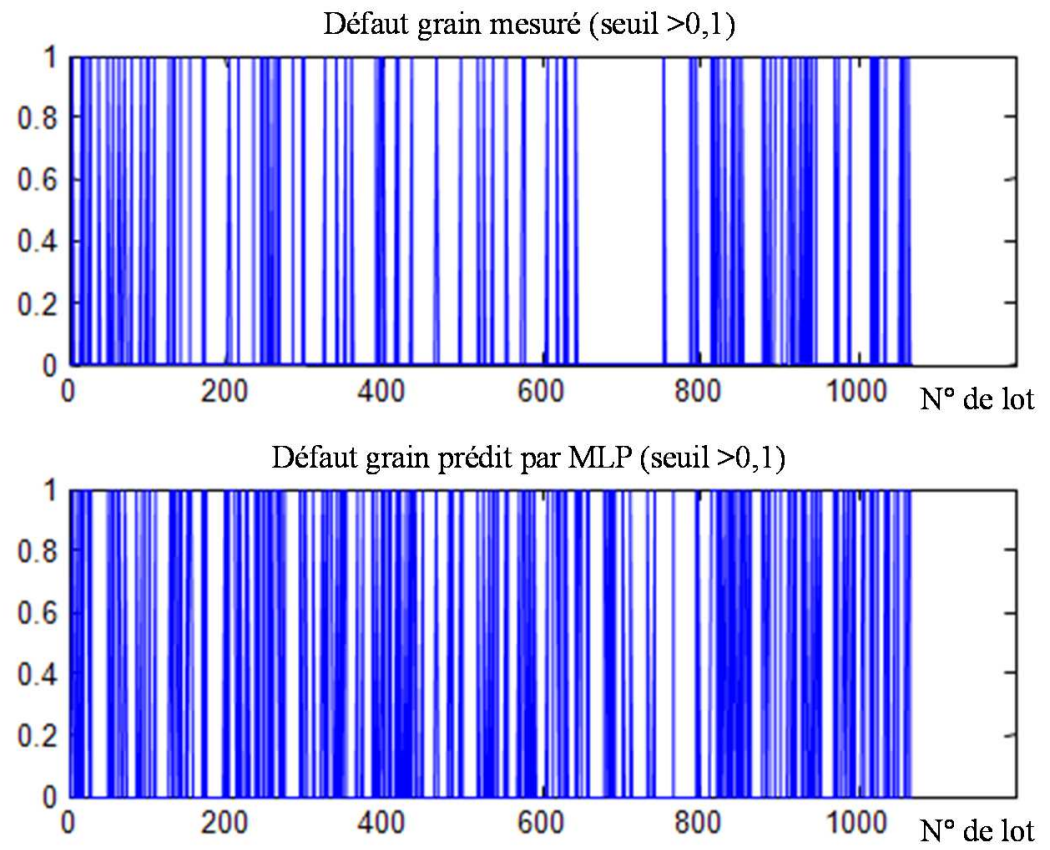

Figure 4. Comparaison des grains relevés à la sortie du robot (en haut) et ceux prédits par le NN (en bas) 
Lors de la phase de validation, les risques de défauts prédits par le modèle et les défauts réellement constatés sont comparés. Sur les 1068 échantillons du jeu de données de validation, 127 correspondent à la présence de défauts «grain ». Sur ces 127 défauts, 112 sont correctement prédits par le modèle ce qui conduit à un taux de non détection de 11,8\%. Par ailleurs, la proportion de «faux positifs » est de 19,2\%. Ce taux important s'explique, pour partie, par la collecte manuelle des défauts en sortie de machine qui n'a pas été correctement menée à bien. Ainsi, la figure 4 présente la comparaison des défauts relevés en sortie de machine et ceux prédits par le modèle.

On constate que pour les lots compris entre les numéros 600 et 800, quasiment aucun défaut n'a été relevé en sortie de robot alors que sur la même période, le modèle en prédit un certain nombre. Ceci est dû au fait que l'opérateur de la machine a été remplacé sur cette période par un intérimaire qui n'a, tout simplement, pas collecté les défauts. Ceci illustre l'importance de la phase de collecte de données qui, si elle n'est pas menée correctement, va inclure dans la base de données des valeurs aberrantes qui vont dégrader les performances du modèle ainsi construit.

Le modèle obtenu nous permet donc de prédire le risque d'occurrence de défauts « grain » sur les produits. Il peut donc être utilisé pour déterminer le réglage optimal des paramètres contrôlables du robot en fonction des caractéristiques du lot considéré et des valeurs des facteurs environnementaux courantes. Pour ce faire, nous utilisons ce modèle en figeant les entrées non contrôlables aux valeurs imposées par l'environnement ou la gamme, et nous simulons un plan d'expériences complet sur les facteurs contrôlables. La figure 5 présente un exemple d'une telle réalisation. Dans cet exemple, le nombre de passes et le nombre de couches sont fixés à 1 . Les autres paramètres non contrôlables sont eux fixés à leurs valeurs médianes. Pour les 3 paramètres contrôlables 10 niveaux ont été choisis ce qui correspond à la simulation de 1000 expériences.
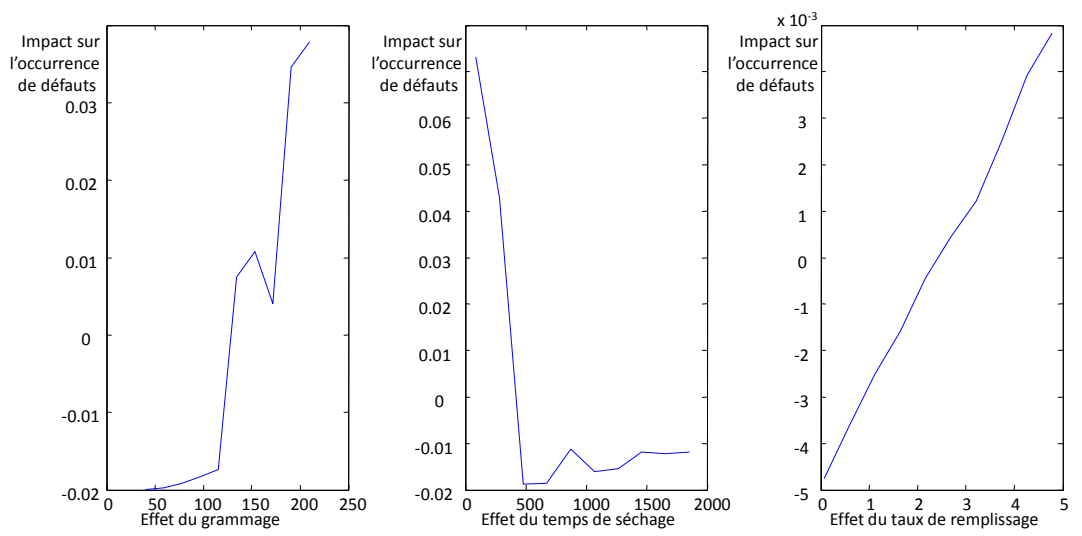

Figure 5. Résultat du plan d'expériences obtenu par simulation sur le modèle

Ces résultats montrent que, pour le lot considéré et dans les conditions environnementales considérées, le taux de remplissage n'a que peu d'impact sur la génération de défauts. Ils permettent également de trouver des bornes de réglage pour les deux autres paramètres. Ainsi, le grammage ne doit pas dépasser 120 (borne supérieure) alors que le temps de séchage ne doit pas être inférieur à 500 (borne inférieure). Il est donc possible de cette manière de régler de manière optimale les paramètres contrôlables en fonction des conditions d'utilisation.

L'utilisation de cette approche a donc permis de réduire l'occurrence de défauts produits au robot de laquage ce qui contribue à diminuer les taux de reprises et donc à simplifier les flux de produits. Cependant, elle n'a pas permis de totalement éradiquer les défauts. Il est donc nécessaire de prendre en compte ces taux de reprises résiduels lors du pilotage de la production.

\subsection{Impact sur le pilotage de la production}

Le taux de reprises résiduel a donc toujours un impact sur les flux de production qu'il est nécessaire d'évaluer afin de pouvoir prendre des décisions. Dans ce but, nous proposons de mettre en œuvre une combinaison d'indicateurs qui nous permettra de construire une cartographie faisant apparaitre différentes zones de fonctionnement de l'atelier, zones auxquelles nous pourrons associer des décisions. Plusieurs combinaisons d'indicateurs ont été proposées dans (Noyel et al. 2014). Nous présenterons uniquement ici la combinaison des deux indicateurs $N_{\text {défaut }}$ (tableau 1) et $N_{\text {retard }}$ (tableau 2). 
Nous avons construit un modèle simplifié de l'atelier considéré à l'aide du logiciel Arena $\odot$ et nous avons fait varier dans ce modèle le taux de reprises et construit les valeurs des deux indicateurs $N_{\text {défaut }}$ et $N_{\text {retard }}$. La figure 6 présente la vue combinée de ces deux indicateurs et fait clairement apparaitre deux seuils de comportement de l'atelier.

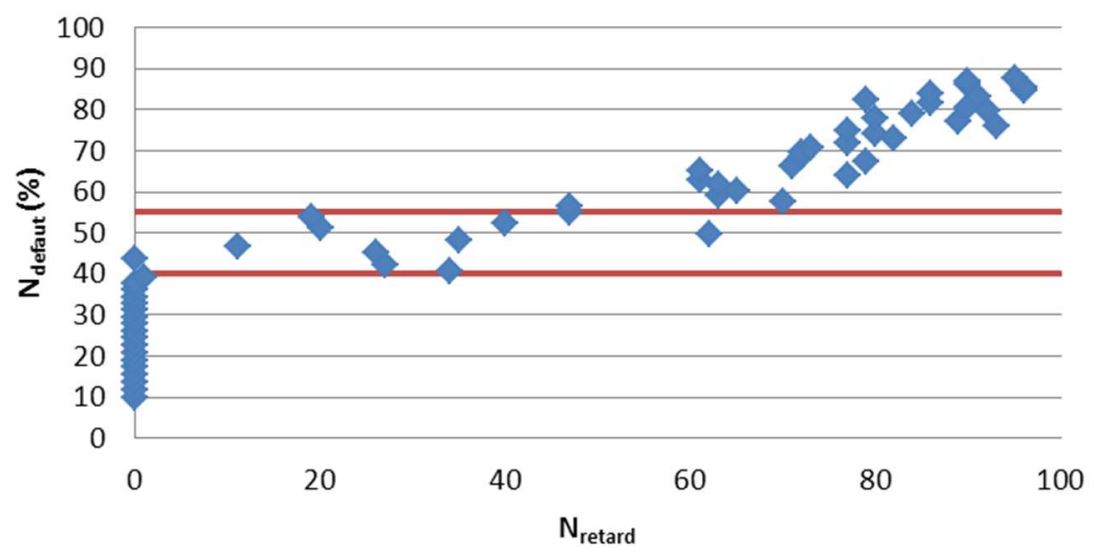

Figure 6. Matérialisation des différentes zones de comportements sur la vue offerte par $N_{\text {retard }}$ et $N_{\text {défaut }}$

Lorsque le taux de reprises augmente, $N_{\text {défaut }}$ augmente puisque chaque reprise correspond à un défaut. Par contre, tant que tant que $N_{\text {défaut }}$ ne dépasse pas un seuil (ici de 40\%) L'indicateur $N_{\text {retard }}$ n'augmente pas car la capacité de l'atelier est suffisante pour absorber cette surcharge de travail. Cependant, dès le franchissement de ce seuil de $40 \%$ une faible augmentation supplémentaire de $N_{\text {défaut }}$ de l'ordre de $5 \%$ va faire exploser le nombre de retard en faisant passer la valeur de cet indicateur de quasiment $0 \%$ à près de $60 \%$. Lorsque le taux de reprises est très haut (points en haut à droite), un même gradient de $5 \%$ de $N_{\text {défaut }}$ ne correspondra plus qu'à un gradient de $15 \%$ de retard. Cette cartographie permet donc bien de faire apparaitre 3 zones de comportement différentes pour l'atelier. La dernière étape qui ne sera pas abordé ici (mais qui fait l'objet de travaux de recherche spécifiques) consiste à déterminer les règles de pilotage et les décisions à prendre lorsque l'atelier se trouve dans l'une ou l'autre de ces zones et ce, afin d'optimiser le pilotage de l'atelier comme expliqué figure 2.

\section{Conclusion}

Ce papier s'intéresse à la réduction de l'impact des taux de reprises sur le pilotage des flux de production. L'approche proposée a pour vocation d'être complémentaire aux philosophies de management de la qualité telle que la TQM et s'articule en deux étapes principales.

La première consiste à réduire les taux de reprises en déterminant le réglage optimal de l'outil de production. Pour cela, un modèle de comportement est construit par extraction de connaissances directement des données opérationnelles dans le but de modéliser le lien entre les facteurs influents et le risque d'occurrence de défauts. Ce modèle est utilisé dans un second temps afin de simuler un plan d'expériences sur les paramètres contrôlables et ainsi d'en déduire un réglage optimal en ligne.

La deuxième étape consiste à évaluer l'impact de ces taux de reprises sur le fonctionnement de l'atelier en utilisant une combinaison d'indicateurs. Cette combinaison d'indicateurs permet d'établir une cartographie du fonctionnement de l'atelier faisant apparaitre différentes zones de comportement auxquelles des règles de pilotage et des décisions peuvent être associées.

L'ensemble de l'approche est testé dans une entreprise de production de panneaux laqués haut de gamme. Les résultats ont montré l'intérêt de l'approche. 
Nos futurs travaux vont plus particulièrement porter sur la détermination automatique des bornes des zones de comportement. Pour ce faire l'utilisation de techniques de clustering semble particulièrement indiquée. Le deuxième point concernera la recherche de la règle de pilotage optimale et donc des décisions à prendre et que l'on devra associer à chacune des zones de fonctionnement établies par la cartographie.

\section{Bibliographie}

Abouzahir O., Gautier R., Gidel T. (2003). Pilotage de l'amélioration des process par les coûts de non-qualité. 10 $0^{\text {ième }}$ Séminaire CONFERE, Belfort, France.

AFGI (1992). Evaluer pour évaluer, les indicateurs de performance au service du pilotage industriel, Association Française de Gestion Industrielle, ouvrage collectif AFGI.

Agard B. and Kusiak A. (2005). Exploration des bases de données industrielles à l'aide du datamining - Perspectives. $9^{\text {ème }}$ colloque national AIP PRIMECA.

Colledani M., Tolio T. (2006). Impact of quality control on production system performance. CIRP Annals-Manufacturing Technology, vol., 55, $\mathrm{n}^{\circ} 1$, p. 453-456.

Crosby P.B. (1979). Quality is Free, Mc Graw-Hill, New-York.

Disney M.S., Towill, D.R. (2003). On the bullwhip and inventory variance produced by an ordering policy. Omega, vol. $31, \mathrm{n}^{\circ}$ 3, p. $157-167$.

Engelbrecht A.P. (2001). A new pruning heuristic based on variance analysis of sensitivity information. IEEE transactions on Neural Networks, p. 1386-1399.

Feigenbaum A.V. (1951). Quality control: Principles, practice and administration: An industrial management tool for improving product quality and design and for reducing operating costs and losses, McGraw-Hill, New-York.

Garvin D. (1984). What does product quality really mean. Sloan management review, vol. 26, n 1, p. 25-48.

Hajek P., Olej V. (2010) Municipal revenue prediction by ensembles of neural networks and support vector machines. WSEAS Transactions on Computers, vol. 9, p. 1255-1264.

Ishikawa K. (1986). Guide to quality control, Asian Productivity Organization, Tokyo.

Kannan V.R., Tan K.C. (2005). Just in time, total quality management, and supply chain management : understanding their linkages and impact on business performance. Omega, vol ; 33, p.153-162.

Love P.E.D., Li H., Mandal P. (1999). Rework: a symptom of a dysfunctional supply chain. European Journal of Purchasing \& Supply Management, vol. 5, $\mathrm{n}^{\circ} 1, \mathrm{p} .1-11$.

Meyer D., Leisch F., Hornik K. (2003). The support vector machine under test. Neurocomputing, vol. 55, p. 169-186.

Nguyen D., Widrow B. (1990). Improving the learning speed of 2-layer neural networks by choosing initial values of the adaptative weights. Proc. of the Int. Joint Conference on Neural Networks IJCNN'90, vol. 3, p. 21-26.

Noyel M., Thomas P., Charpentier P., Thomas A., Brault T. (2013). Implantation of an on-line quality process monitoring. $5^{\text {th }}$ International Conference on Industrial Engineering and Systems Management IESM'13, Rabat, Maroc.

Noyel M., Thomas P., Charpentier P., Thomas A., Brault T. (2014). Flow disturbance analysis in workshops with high reworks rate. Advances in Production Management Systems APMS'14, Ajaccio, France.

Noyel M. (2015). Contrôle intégré du pilotage d'atelier et de la qualité des produits. Application à la société ACTA mobilier. Thèse de l'Université de Lorraine.

Paliwal M., Kumar U.A. (2009). Neural networks and statistical techniques: A review of applications. Expert Systems with Applications, vol. 36, p. 2-17.

Patel M.C., Panchal M. (2012). A review on ensemble of diverse artificial neural networks. Int. J. of Advanced Research in Computer Engineering and Technology, vol. 1, $\mathrm{n}^{\circ}$ 10, p. 63-70.

Setiono R., Leow W.K. (2000). Pruned neural networks for regression. $6^{\text {th }}$ Pacific RIM Int. Conf. on Artificial Intelligence PRICAI'O0, Melbourne, Australia.

Taguchi, G. (1989). Quality Engineering in Production Systems, Mc Graw-Hill, New-York.

Talib F., Rahman Z. (2012). Total quality management practices in manufacturing and service industries: a comparative study. Int. J. Advanced Operations Management vol. 4, $\mathrm{n}^{\circ}$ 3, p. 155-176.

Thomas P., Bloch G. (1997). Initialization of one hidden layer feedforward neural networks for non-linear system identification. $15^{\text {th }}$ IMACS World Congress on Scientific Computation, Modelling and Applied Mathematics WC'97, Berlin, Allemagne.

Thomas P., Bloch G., Sirou F., Eustache V. (1999). Neural modeling of an induction furnace using robust learning criteria. $J$. of Integrated Computer Aided Engineering, vol. 6, $\mathrm{n}^{\circ}$ 1, p. 5-23. 
Thomas P., Noyel M., Suhner M.C., Charpentier P., Thomas A. (2013). Neural Networks ensemble for quality monitoring. $5^{\text {th }}$ International Joint Conference on Computational Intelligence IJCCI'13, Vilamoura, Portugal.

Thomas P., Suhner M.C., (2015). A new multilayer perceptron pruning algorithm for classification and regression applications. Neural Processing Letters., vol. $42, \mathrm{n}^{\circ} 2$, p. 437-458.

Vollmann T.E., Berry W.L., Whybark C.D. (1984). Manufacturing Planning and Control Systems, Dow Jones-Irwin, NewYork. 